\title{
Mejora de las propiedades termomecánicas de grafito isótropo, elaborado a partir de mesofase carbonosa, mediante la adición de carburos
}

\author{
N. ORDÁS' , J. ECHEBERRIA', M. BALDEN², S. LINDIG², C. GARCÍA-ROSALES'1 \\ ${ }^{1}$ Centro de Estudios e Investigaciones Técnicas de Gipuzkoa and Escuela Superior de Ingenieros, Universidad de Navarra.20009 San Sebastián, Spain \\ ${ }^{2}$ Max-Planck-Institut für Plasmaphysik, EURATOM Association, D-85748 Garching, Germany
}

\begin{abstract}
Este trabajo muestra la posibilidad de desarrollar materiales grafíticos isótropos dopados para su implantación en las zonas de mayor flujo térmico de la primera pared de futuros experimentos de fusión nuclear. Estos materiales deben tener elevada conductividad térmica y alta resistencia al choque térmico. Como material de partida se han empleado mezclas de mesofase carbonosa y diferentes carburos metálicos $\left(\mathrm{TiC}, \mathrm{VC}, \mathrm{ZrC}, \mathrm{WC}\right.$ y $\mathrm{B}_{4} \mathrm{C}$ ). Estos carburos catalizan la grafitización, permitiendo que tenga lugar a menor temperatura. VC comienza a actuar como catalizador a la temperatura más baja; $\mathrm{ZrC}$ es el catalizador más eficaz de los estudiados. Se ha observado que al grafitizar a la temperatura a la que comienza el efecto catalítico de los carburos, la porosidad abierta deja de estar conectada. Este fenómeno sugiere la existencia de un mismo mecanismo de difusión responsable del efecto catalítico y del cierre de la porosidad abierta. La adición de carburos no afecta negativamente a las propiedades mecánicas.
\end{abstract}

Palabras clave: grafitos dopados, mesofase, catalisis de la grafitización,

Improvement of the thermo-mechanical properties of isotropic graphite, produced from carbonaceous mesophase, by the addition of different carbides

This work demonstrates the possibilities for optimization of doped fine grain graphites with high thermal conductivity and high thermal shock resistance for application as plasma-facing materials at those areas of the vessel wall in fusion devices receiving the highest particle and power loads. A mixture of carbonaceous mesophase powder and different fine grain carbides $\left(\mathrm{B}_{4} \mathrm{C}, \mathrm{TiC}, \mathrm{VC}, \mathrm{ZrC}\right.$ and $\left.\mathrm{WC}\right)$ was used as starting material. These carbides act as catalysts, decreasing the graphitization temperature. VC acts as catalyst of the graphitization at the lowest temperature, and $\mathrm{ZrC}$ is the most effective catalyst of all investigated carbides. With increasing graphitization temperature, the open porosity of all doped materials becomes closed, suggesting the existence of a diffusion mechanism responsible for both the catalytic effect and the closing of the open porosity. Carbides addition does not strongly influence the mechanical properties of pure graphite.

Keywords: doped graphite, mesophase, catalytic graphitization

\section{INTRODUCCIÓN}

Los materiales basados en carbono son candidatos para su aplicación como material de recubrimiento en la primera pared de vasijas de futuros reactores de fusión nuclear, en aquellas zonas que van a quedar expuestas a mayores cargas térmicas y de partículas. Uno de los puntos críticos en este tipo de aplicaciones es la erosión química debida al bombardeo por hidrógeno proveniente del plasma $(1,2)$, y la retención de tritio. Se sabe que la adición de pequeñas cantidades de algunos elementos como $\mathrm{B}, \mathrm{Si}$, Ti o $\mathrm{W}$ contribuye a reducir la erosión química (3). Por otra parte, es necesario que estos materiales presenten una elevada conductividad térmica para permitir una rápida transferencia de calor, y una elevada resistencia al choque térmico (alta resistencia mecánica y bajo módulo de Young).

El objetivo de este trabajo es la obtención de grafito isótropo de grano fino, dopado con carburos, con una reducida erosión química, elevada conductividad térmica y alta resistencia al choque térmico, para hacerlo competitivo con otros materiales candidatos para la primera pared de reactores de fusión.

\section{PARTE EXPERIMENTAL}

Como material de partida se ha utilizado MCMB (mesocarbon microbeads $=$ microesferas de mesofase carbonosa) obtenidas a partir de residuos de petróleo en una planta semiindustrial de la compañía REPSOL-YPF. Tras ser micronizadas presentaban un tamaño de partí- cula medio de $2.6 \mu \mathrm{m}$. Como dopantes se han utilizado $\mathrm{B}_{4} \mathrm{C}, \mathrm{TiC}, \mathrm{V}_{8} \mathrm{C}_{7^{\prime}}$ WC y ZrC, en una proporción del $4 \%$ atómico del metal de cada carburo. Todos ellos actúan como catalizadores de la grafitización (4), son estables a temperaturas muy elevadas, tienen una presión de vapor relativamente baja, y la mayoría presentan un rango de estequiometría relativamente ancho $(5,6)$. Su tamaño medio de partícula se encuentra alrededor de $1 \mu \mathrm{m}$, excepto para el WC, que es de $200 \mathrm{~nm}$.

Tras la compactación, las muestras fueron calcinadas a $1000^{\circ} \mathrm{C}$ en atmósfera de $\mathrm{N}_{2}$ a bajas velocidades de calentamiento, para permitir la salida de volátiles sin que se produzca agrietamiento de los compactos. La grafitización se llevó a cabo en atmósfera de He a temperaturas de hasta $2900^{\circ} \mathrm{C}$

Tras la grafitización, la altura de los cristalitos de grafito en la dirección perpendicular a los planos basales, $L_{c^{\prime}}$ fue medida mediante difracción de rayos- $X$ a partir de la reflexión del plano basal (002).

La conductividad térmica para las distintas muestras fue determinada a partir de la difusividad térmica, la densidad y la capacidad calorífica. La capacidad calorífica fue obtenida de la literatura (7), teniendo en cuenta la concentración de carburo para cada muestra. La difusividad térmica tras la grafitización fue determinada mediante el método laser-flash en el rango de temperaturas $20-1200^{\circ} \mathrm{C}$.

La resistencia a la flexión, el módulo de Young y la deformación a fractura fueron obtenidos a partir de ensayos de flexión en tres puntos. Se utilizaron 4 probetas para cada material. 


\section{RESULTADOS Y DISCUSIÓN}

La Fig. 1 muestra el tamaño de los cristalitos de grafito $\left(L_{c}\right)$ para distintas temperaturas de grafitización, bajo las mismas condiciones (velocidad de calentamiento $10^{\circ} \mathrm{C} / \mathrm{min}, 1 \mathrm{~h}$ mantenimiento), excepto para el ciclo a $2900^{\circ} \mathrm{C}$, en el que no hubo mantenimiento a la máxima temperatura. Como se puede observar, los carburos no catalizan la grafitización por debajo de una determinada temperatura, distinta para cada carburo: el tamaño de los cristalitos apenas varía fuera del rango de temperaturas en el que se produce la grafitización catalítica. Esta temperatura parece estar relacionada con la temperatura de eutéctico del carburo (ver Fig. 1). VC actúa como catalizador a la temperatura más baja, dando lugar a un repentino aumento de $L_{c^{\prime}}$ en un rango de temperaturas relativamente estrecho (2500-2600). $\mathrm{ZrC}$ es el catalizador más efectivo, puesto que permite conseguir un mayor aumento en $L_{c}$ respecto del material sin dopar. La disminución de $L_{c}$ en las muestras con $\mathrm{ZrC}$ grafitizadas a $2900^{\circ} \mathrm{C}$ es debida probablemente a que no hubo mantenimiento a la temperatura máxima.

Se ha representado la conductividad térmica a temperatura ambiente de distintas muestras grafitizadas, en función del tamaño de los cristales de grafito, $L_{\mathrm{c}}$ para comprobar si existe una relación entre ambos parámetros (Fig. 2). Se han incluido muestras procedentes de tres ciclos distintos de grafitización $\left(2710^{\circ} \mathrm{C}\right.$, mantenimiento 5 min.; $2750^{\circ} \mathrm{C}$ mantenimiento $1 \mathrm{~h} ; 2900^{\circ} \mathrm{C}$ sin mantenimiento) elaboradas con dos materiales de partida distintos: MCMB procedente de REPSOL (R) y MCMB producida a escala de laboratorio en la Universidad de Alicante (A1 y A2). Se observa claramente una relación lineal para todos los materiales, excepto para las muestras dopadas con $\mathrm{B}_{4} \mathrm{C}$ (R-B) y con $\mathrm{ZrC}$ (R-Zr). En el primer caso, la desviación de la relación lineal es debida a que la solubilidad substitucional del B en la red del grafito (hasta $2.35 \%$ atómico a $2350^{\circ} \mathrm{C}(8)$ ) incrementa la dispersión por fonones y, por tanto, reduce la conductividad térmica, contrarrestando el efecto catalítico del carburo. En las muestras dopadas con $\mathrm{ZrC}$, el máximo de la conductividad parece estar a temperaturas por debajo de temperatura ambiente (9), lo que explicaría que ya no hubiese una dependencia lineal con el tamaño de los cristalitos, ya que empieza a dominar la dispersión por fonones.

Se ha estudiado la porosidad de las muestras elaboradas con MCMB de Repsol tras la grafitización a distintas temperaturas, manteniendo constantes el resto de parámetros del tratamiento. Como se muestra en la Fig. 3, en el rango de temperaturas $1000-1550^{\circ} \mathrm{C}$, correspondiente a la transformación de carbono desordenado a turbostrático, la porosidad total de todas las muestras disminuye, ya que se produce sinterización en fase sólida. La menor disminución observada tanto para las muestras dopadas con $\mathrm{VC}$ como con $\mathrm{ZrC}$ puede deberse a la reducción de los óxidos que se habían formado durante la calcinación, o que ya estaban presentes en el polvo de carburo de partida: la salida de oxígeno del material deja tras de sí porosidad adicional. Para temperaturas de grafitización mayores de $\sim 1500^{\circ} \mathrm{C}$ la porosidad total de todas las muestras permanece prácticamente constante.

Sin embargo, mientras la porosidad abierta y cerrada de las muestras sin dopar permanecen constantes al aumentar la temperatura de grafitización, la porosidad abierta de las muestras dopadas se transforma en cerrada, sin que haya densificación del material. En los ciclos en los que la temperatura ha superado la correspondiente a la grafitización catalítica de cada material, prácticamente toda la porosidad es cerrada, pese a que la porosidad total es notable ( $>10 \%$ vol). Estas observaciones sugieren que el mismo mecanismo de difusión que permite que los carburos actúen como catalizadores de la grafitización va cerrando la porosidad abierta. Esta observación se ve respaldada por el hecho de que la evolución de la porosidad se produce en el mismo rango de temperaturas que el rápido crecimiento de los cristales de grafito (ver Fig. 1 y Fig. 3).

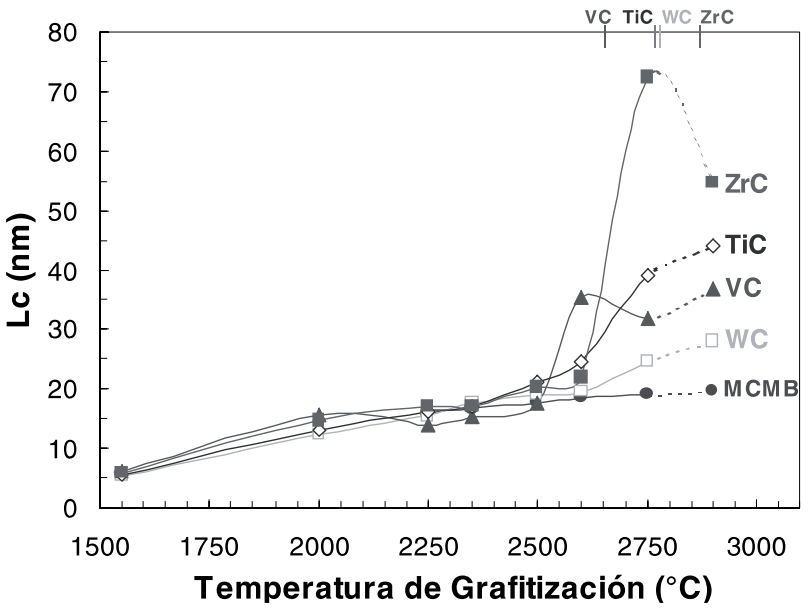

Fig. 1. Tamaño de los cristalitos, $L_{c^{\prime}}$ en función de la temperatura de grafitización para la muestra sin dopar (MCMB) y las muestras dopadas con carburos. Se indican las temperaturas de los eutécticos.

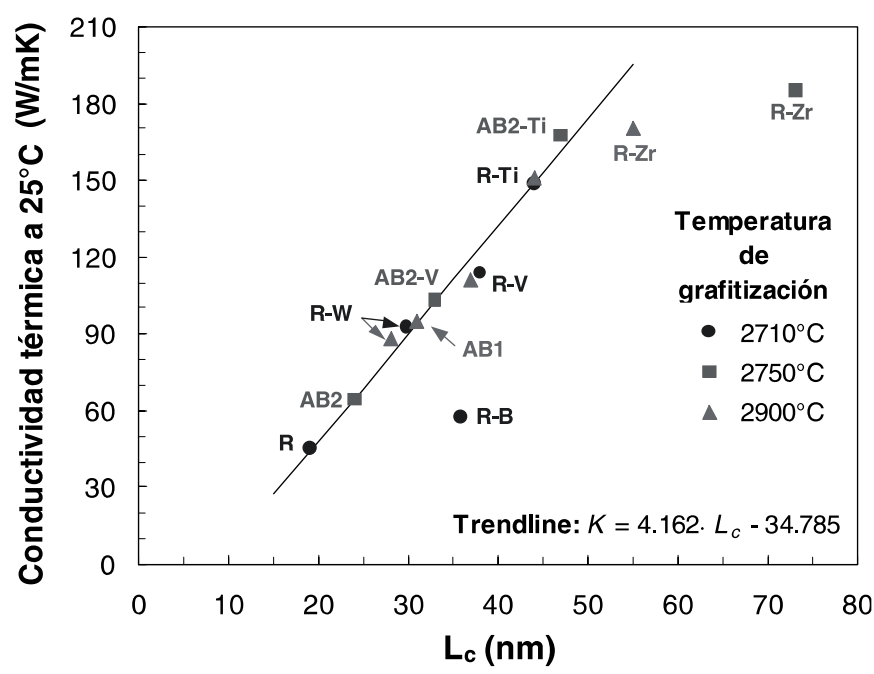

Fig. 2. Conductividad térmica a temperatura ambiente en función del tamaño de los cristalitos, $L_{c^{\prime}}$ tras distintos ciclos de grafitización. Los dopantes se indican tras el guión.

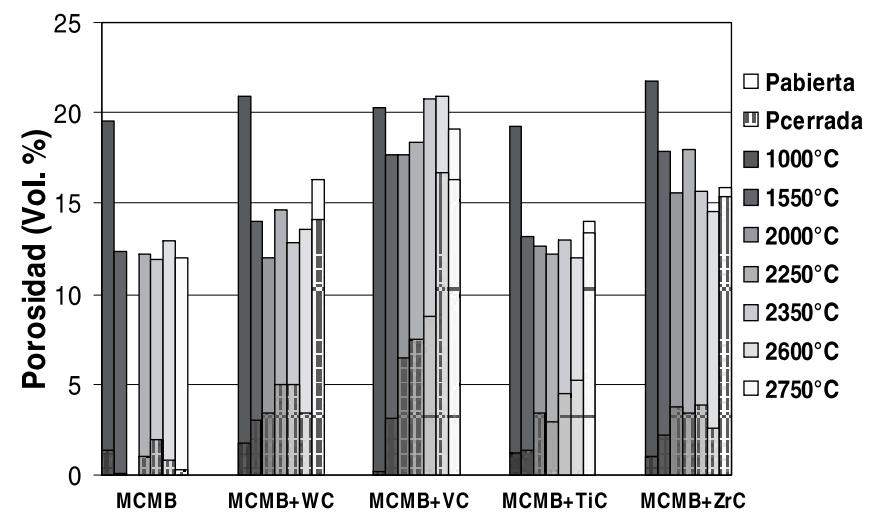

Fig. 3. Porosidad abierta y cerrada para las muestras grafitizadas.

Se sometieron diversas muestras a un ciclo de HIP (Hot Isostatic Pressing) tras la grafitización y, como se puede ver en la Fig. 4, en las muestras dopadas la porosidad se redujo notablemente, especialmente en aquellas dopadas con WC, en las que se ha conseguido una porosidad menor del $3 \%$ en volumen. Esto indica que, efectivamente, la grafitización catalítica activa una serie de mecanismos que estrangu- 
lan u obstruyen los poros, impidiendo que éstos estén conectados con la superficie.

Se realizaron ensayos de flexión en 3 puntos para estudiar las propiedades mecánicas: módulo de Young $(E)$, resistencia a la flexión $(\sigma)$, deformación a fractura $\left(\varepsilon_{f}\right)$ y relación $\sigma_{f} / E$. Los resultados se muestran en la Tabla 1, tanto para las muestras sólo grafitizadas (G) como para las sometidas a un ciclo de $\operatorname{HIP}\left(1900^{\circ} \mathrm{C}, 200 \mathrm{MPa}\right)$ tras la grafitización (GH). El material sin dopar presenta mejores propiedades mecánicas que la mayoría de grafitos comerciales de grano fino (10). La adición de carburos no parece influir negativamente en las propiedades mecánicas, y en algunos casos (MCMB+VC) las mejora notablemente, ya que se produce un significativo aumento en $\sigma / E$. Los valores de deformación a fractura y resistencia a la flexión de las muestras dopadas con TiC grafitizadas y sometidas a HIP no son fiables, puesto que presentaban grietas previas a la flexión. Para el resto de materiales, se ha comprobado que mediante HIP se conservan o mejoran todas las propiedades mecánicas.

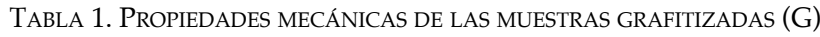
ANTES DE HIP (GH) TRAS HIP.

\begin{tabular}{|c|c|c|c|c|c|c|}
\hline & \multirow[b]{2}{*}{ Trat. } & \multicolumn{5}{|c|}{ Material } \\
\hline & & MCMB & $\begin{array}{c}\mathrm{MCMB} \\
+ \\
\mathrm{TiC}\end{array}$ & $\begin{array}{c}\text { MCMB } \\
+ \\
\mathrm{VC}\end{array}$ & $\begin{array}{c}\mathrm{MCMB} \\
+ \\
\text { WC }\end{array}$ & $\begin{array}{c}\mathrm{MCMB} \\
+ \\
\mathrm{ZrC}\end{array}$ \\
\hline \multirow{2}{*}{$\begin{array}{l}\mathrm{E} \\
\mathrm{GPa}\end{array}$} & G & 6.4 & 5.8 & 4.8 & 6.2 & 6.3 \\
\hline & GH & 7.2 & 5.7 & 3.1 & 6.5 & 7.1 \\
\hline \multirow{2}{*}{$\sigma_{\mathrm{f}} \mathrm{MPa}$} & G & 93 & 105 & 105 & 108 & 122 \\
\hline & GH & 105 & 75 & 98 & 121 & 127 \\
\hline \multirow{2}{*}{$\varepsilon_{\mathrm{f}} \%$} & G & 1.39 & 1.56 & 2.05 & 1.73 & 1.73 \\
\hline & GH & 1.45 & 1.35 & 3.08 & 1.84 & 1.95 \\
\hline \multirow{2}{*}{$\sigma_{f} / E \times 10^{-3}$} & G & $\begin{array}{l}14.5 \\
\end{array}$ & 15.6 & 22.0 & 17.6 & 16.7 \\
\hline & GH & 14.6 & 13.3 & 31.4 & 18.6 & 16.2 \\
\hline
\end{tabular}

\section{RESUMEN}

Las conclusiones más relevantes del presente trabajo son las siguientes:

- VC actúa como catalizador a la temperatura de grafitización más baja, seguido de TiC, WC y ZrC. Esta secuencia es la misma que la correspondiente a la temperatura del eutéctico. $\mathrm{ZrC}$ es el catalizador de la grafitización más efectivo de todos los carburos estudiados.

- $L_{c}$ es directamente proporcional a la conductividad térmica a temperatura ambiente para todos los materiales, excepto para los dopados con $\mathrm{B}_{4} \mathrm{C}$ (solubilidad del $\mathrm{B}$ en la red del grafito) o con $\mathrm{ZrC}$ (mayor contribución de la dispersión por fonones).

- Un aumento de la temperatura de grafitización por encima de un valor determinado, distinto para cada carburo, conlleva una transformación de la porosidad abierta a cerrada, sin que haya densificación del material. Esta evolución coincide con la dependencia observada entre $L_{c}$ y la temperatura de grafitización, y tiene lugar a temperaturas cercanas la correspondiente a la grafitización catalítica para cada material. Este fenómeno sugiere la existencia de un mecanismo de difusión responsable del efecto catalítico de la grafitización y del cierre de la porosidad abierta.

- El uso de MCMB como material de partida, y el empleo de HIP se presentan como una ruta eficaz para la obtención de grafitos dopados de alta densidad, sin necesidad de recurrir a ligantes durante su elaboración.

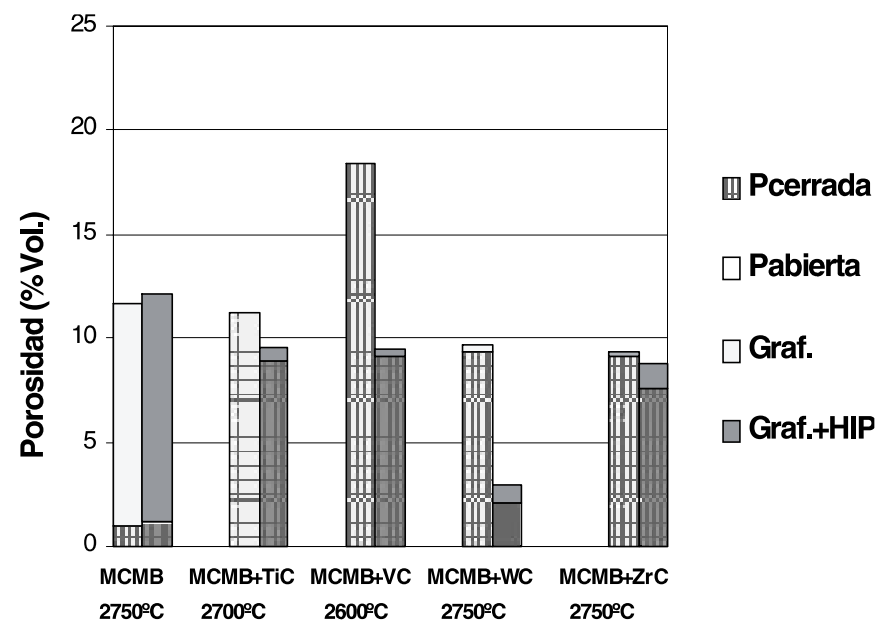

Fig. 4. Porosidad abierta y cerrada para muestras grafitizadas antes y después de HIP.

- La relación resistencia a la flexión/módulo de Young y la deformación a fractura son mayores que para la mayoría de los grafitos comerciales. Las propiedades mecánicas no se ven afectadas negativamente por la adición de carburos. Mediante HIP se consiguen ligeras mejoras de las propiedades mecánicas.

\section{AGRADECIMIENTOS}

Los autores agradecen a REPSOL-YPF y M. Martínez Escandell (Departamento de Química Inorgánica, Universidad de Alicante) por proporcionar la mesofase de partida. Asimismo agradecen la financiación a la CICYT (proyecto FTN2000-0915-C03-02) así como a la EFDA Technology Workprogramme 2000 (Task T438) a través de la asociación EURATOM Ciemat.

\section{BIBLIOGRAFÍA}

1. J. Roth, C. García-Rosales. "Analytic description of the chemical erosion of graphite by hydrogen ions". Nucl. Fus. 361647 (1996). With corrigendum Nucl. Fus. 37897 (1997).

2. E. Vietzke, A.A. Haasz. "Chemical erosion”, pp. 135-176, en Physical Processes of the Interaction of Fusion Plasmas with Solids. Eds. W.O. Hofer, J. Roth, Academic Press, San Diego, (EE.UU.) 1996.

3. C. García-Rosales, M. Balden. "Chemical erosion of doped graphites for fusion devices". J. Nucl. Mater. 290-293, 173-179 (2001)

4. A. ya, H. Marsh. "Review - Phenomena of catalytic graphitization". J. Mater. Sci. 17309 (1982).

5. T.B. Massalski. Binary Alloy Phase Diagrams vol. 1. American Society for Metals, 1986.

6. J. Gurland. Int. Mat. Rev. 33151 (1988).

7. O. Knacke, O. Kubaschewski, K. Hesselmann (Eds.). Thermochemical Properties of Inorganic Substances, $2^{\text {nd }}$ Ed., Springer-Verlag, Verlag Stahleisen m.b.H. Düsseldorf, 1991.

8. C.E. Lowell. J. Am. Ceram. Soc. 50142 (1967)

9. C. García-Rosales, N. Ordás, E. Oyarzabal, J. Echeberria, M. Balden, S. Lin$\mathrm{dig}$, R. Behrisch. "Improvement of the thermo-mechanical properties of fine grain graphite by doping with different carbides", aceptado para su publicación en J. Nucl. Mater.

10. W. Delle, J. Linke, H. Nickel, E. Wallura, “Comparison of high purity fine grain graphites from different suppliers with regard to physical, mechanical and thermal properties", Jül-Spez-401, Forschungszentrum Jülich, 1987.

Recibido: 1.2 .03

Aceptado: 30.11 .03 